\title{
Status Epilepticus in the PICU: Quieting the Storm
}

\author{
David T. Hsieh $\cdot$ Elizabeth A. Thiele
}

Published online: 8 January 2013

(c) Springer Science + Business Media New York 2013

\begin{abstract}
Status epilepticus (SE), defined by either a prolonged seizure or a prolonged period of recurrent seizures without a return to baseline in-between seizures, is a neurological emergency frequently encountered in the pediatric intensive care unit (PICU). Pediatric SE, in addition to itself leading to PICU admission, occurs commonly in critically ill children. The clinical manifestations of SE are increasingly being recognized as presenting in a widely heterogeneous manner, particularly in the PICU. Subtler presentations can include altered mental status with or without suggestive motor movements. Thus, the initial fundamental task in managing pediatric SE is astute recognition and diagnosis, which often requires both clinical acumen and electroencephalography. This, in turn, allows for prompt management with concurrent evaluation and treatment. In this article, we will review the diagnosis, evaluation, and treatment of SE occurring in the setting of the PICU, with a focus on bringing the reader up-to-date on these topics.
\end{abstract}

Keywords Electroencephalography - Status epilepticus · Intensive care units · Pediatric

\section{T. Hsieh}

Division of Child Neurology, Department of Pediatrics,

San Antonio Military Medical Center, 3551 Roger Brooke Dr,

Ft Sam Houston, TX 78234, USA

e-mail: david.hsieh@us.af.mil

\section{E. A. Thiele $(\square)$}

Pediatric Epilepsy Program, Department of Neurology,

Massachusetts General Hospital, 175 Cambridge Street,

Suite 340, Boston, MA 02114-2796, USA

e-mail: ethiele@partners.org

\section{Introduction}

Status epilepticus (SE) is a neurological emergency defined by either a prolonged seizure or a prolonged period of recurrent seizures without a return to baseline in-between seizures. Pediatric SE, in addition to itself being a neurological emergency leading to admission and requiring intensive care, occurs commonly in critically ill children, and is therefore frequently encountered in the pediatric intensive care unit (PICU) [1]. Although the analogy of a "storm" may invoke the visual scenario of a child in a continuous generalized tonic-clonic seizure, the clinical manifestations of SE are increasingly becoming recognized to present in a widely heterogeneous manner, particularly in the PICU. Subtler presentations of SE in the PICU, due in part to the common use of sedative and neuromuscularblocking agents in the setting of critically ill children, can include altered mental status with or without suggestive motor movements. Thus, the initial fundamental task in managing pediatric SE- "quieting the storm"-is astute recognition and diagnosis, which in the PICU often requires both clinical acumen and neurophysiological monitoring with electroencephalography (EEG). This, in turn, allows for prompt management with concurrent evaluation and treatment.

\section{Defining Pediatric Status Epilepticus}

The classical definition of SE from the International League Against Epilepsy (ILAE) [2] establishes a cut-off of a duration greater than $30 \mathrm{~min}$. This temporally based definition was influenced by evidence in animal studies establishing the relationship between prolonged seizures and neuronal injury [3]. The onset of seizures, however, is 
not always directly witnessed, which often affects the precision of this definition. Furthermore, advocacy for earlier aggressive treatment has resulted in an evolving definition of SE.

Shorter durations of seizures, 5-10 min, have subsequently been advocated to be the defining standards for SE. The reasons for this are that the vast majority $(>90 \%)$ of seizures last under $2 \mathrm{~min}$ [4], and seizures lasting over 5-10 min are unlikely to stop without medical intervention [5]. Furthermore, the need for urgency in treatment is supported by data in adults that establishes a lower initial response rate in patients for whom treatment of SE is delayed [6]. Thus, in clinical practice, using shorter durations for defining SE frames an implied sense of urgency for more aggressive treatment. The first $10-30 \mathrm{~min}$ can also be referred to as early or impending SE, and $30 \mathrm{~min}$ as established SE. The importance of classifying seizures lasting greater than $5 \mathrm{~min}$ as $\mathrm{SE}$ is to underscore the importance of early aggressive treatment [7].

\section{Diagnosis of Status Epilepticus}

Within the PICU, children with SE generally present in one of the following scenarios: either a patient is diagnosed with SE and is being admitted to the PICU for management, or a patient admitted for an alternative clinical diagnosis develops or is diagnosed with SE while in the PICU. The recognition of SE is complicated by its varied semiological presentations. SE can occur for all seizure semiologies as defined by the ILAE [8], for example, simple complex partial SE or complex partial SE, and for generalized seizures as tonic, clonic, tonic-clonic, or myoclonic SE. In such cases, the diagnosis of SE can often be made clinically. It is also important to realize that intermittent seizures without a return to baseline in-between is also $\mathrm{SE}$, as under-recognition often results in delays in medical care [9].

The use of continuous EEG monitoring is now common in intensive care units [10] and complements clinical acumen to diagnose nonconvulsive seizures (NCS) and nonconvulsive status epilepticus (NCSE). With the more common use of continuous EEG, it has been increasingly recognized that pediatric SE can present with a wide range of manifestations, from coma to milder, more intermittent presentations of altered mental status, with or without obvious motor manifestations.

Several recent studies have evaluated for the prevalence of NCS or NCSE among critically ill children using EEG (Table 1). The majority of these studies are retrospective, and have found either NCS or NCSE in 16-39\% of patients, and NCSE in $0-35 \%$ of patients [11-17]. In addition, there have been three recent prospective studies, for which either NCS or NCSE was diagnosed in critically ill children using
Table 1 Nonconvulsive seizures (NCS) or nonconvulsive status epilepticus (NCSE) in critically ill children

NCS or NCSE

Retrospective data: 16-39\% [11, 15-17]

Prospective data: $7-46 \%\left[18,19,20^{\bullet}\right]$

NCSE

Retrospective data: $0-35 \%$ [11-15, 17]

Prospective data: $1-19 \%[18,19,20 \bullet]$

Clinical or electrographic risk factors for NCS or NCSE:

Younger age ( $<24$ months) [12, 17, 19, 20•]

Clinical seizure prior to EEG $[11,14,16-19]$

Epileptiform discharges on EEG [11, 16, 17]

TBI [17]

Duration of EEG to detect NCS or NCSE:

Within the first 30-60 min: 43-71\% [11, 17-19, 20•]

Within the first $24 \mathrm{~h}: 80-100 \%$ [11, 17-19, 20•]

$E E G$ electroencephalogram, $T B I$ traumatic brain injury

EEG in $7 \%$ [18], $30 \%$ [19], or $46 \%$ [20•], with NCSE diagnosed in 1 [18], 18 [19], and 19 [20•]. Some possible explanations for these wide variations in reported ranges include differences in ICU populations and ascertainment of EEG monitoring. For example, $90 \%$ of the patients in the series from Abend et al. [20•] had primary brain disorders, whereas about half of the patients in the series from Shahwan et al. [18] had primary brain disorders. In terms of ascertainment of EEG monitoring, Schreiber et al. [19] used inclusion criteria of a Glasgow coma scale $<12$ not explained by medication or baseline chronic encephalopathy, whereas Abend et al. [20 ] used differing criteria: persisting altered mental status after a convulsion, or altered mental status of unclear etiology or "disproportionate to the known medical condition", and the clinical suggestion of seizures (vital sign changes). In the series from Saengpattrachai, $16 \%$ were diagnosed with NCS, while no patients were diagnosed with NCSE; however, in this series continuous long-term EEG was not utilized [15]. Despite these wide ranges, it can be summarized that critically ill children are at risk to have NCS and NCSE.

Some reported risk factors for NCS or NCSE include younger age $(<2$ years) $[12,17,19,20 \bullet]$, and a clinical seizure prior to EEG monitoring [11, 14, 16-19]. In the series from Abend et al. [20•] for every 1-year increase in age, there was a $7 \%$ decrease in the odds of seizure occurrence. Traumatic brain injury was identified as an independent risk factor in the series from Williams et al. [17]. In addition, in a series looking specifically at children treated with hypothermia after cardiac arrest, NCS were diagnosed with continuous EEG in 47, and $32 \%$ with NCSE [21]. It should also be noted that NCS and NCSE can also occur in other inpatient units outside of the PICU in children with altered mental status, which may require 
transfer to the PICU for management. In a recent study more than half of the patients with altered mental status who were found to be in NCSE were not critically ill [22•].

As predictors for differentiating between ill patients with NCS versus those in NCSE have not been identified, continuous EEG is needed when either NCS or NCSE are suspected. In terms of duration of EEG monitoring, one must balance the yield of diagnosing NCS or NCSE versus the resources available to support continuous EEG. The first NCS is noted within the first 30-60 min of monitoring in $43-71 \%$ and within the first $24 \mathrm{~h}$ in $80-100 \%$ [11, 17-19, 20•]. Thus extension of continuous EEG monitoring to at least $24 \mathrm{~h}$ improves the yield. Finally, although rare in children, the possibility of psychogenic non-epileptic seizures (PNES) resembling convulsive SE has been reported [23]. Diagnosing PNES requires EEG as part of the clinical evaluation and should be utilized in patients with an atypical history or clinical course. Continuous EEG often alters treatment [24] and is the only reliable way to diagnose NCSE in critically ill children with altered mental status. Continuous EEG and child neurology consultation should be strongly considered for patients with suspected NCS or NCSE. Once a child has been identified to be in SE, concurrent evaluation and pharmacological treatment should commence.

\section{Evaluation of Status Epilepticus}

It is important to understand the etiologies of pediatric SE, as this influences the clinical evaluation. The etiology of pediatric SE is classified most commonly into the following categories:

- Febrile SE is provoked by fever in the absence of a central nervous system infection, and typically occurs within the age range of 6 months to 5 years.

- Acute symptomatic SE occurs in the setting of an acute provoking factor, excluding prolonged febrile convulsions. Examples include central nervous system infections, trauma, and stroke.

- Remote symptomatic SE occurs in patients with a prior neurological abnormality without an acute provoking factor. Examples include cerebral dysgenesis and remote brain injury.

- Cryptogenic SE occurs in the absence of a definable cause.

- Idiopathic SE occurs in patients with an idiopathic genetic predisposition for epilepsy.

- SE due to progressive encephalopathy occurs in neurodegenerative disorders.

In prior retrospective studies evaluating for etiologies of pediatric SE [25, 26], the most common etiology is febrile
SE, occurring in about a third of patients. This is followed by remote symptomatic (12-28\%), acute symptomatic (17-18\%), with the remainder classified as either idiopathic or cryptogenic (11-12\%), or progressive neurological $(4 \%)[25,26]$. The age of the patient influences the etiology, as younger children are more prone to present with febrile SE [27] and due to acute symptomatic etiologies, in contrast to older children who tend to present in SE from cryptogenic or remote symptomatic etiologies [28].

About $10 \%$ of children presenting in SE have no history of seizures [5, 29•]. A recent study from Singh et al. [29•] specifically evaluated the scenario in which a child who has no history of seizures presents in SE, using prospectively obtained data. Singh et al. found that one-third of these cases were due to febrile SE, but cryptogenic SE made up the majority of cases, at $42 \%$, with remote symptomatic at $18 \%$, acute symptomatic at $16 \%$, and idiopathic at $4 \%$ of cases. [29•] Although the authors defined SE at $20 \mathrm{~min}$ and also excluded patients with prolonged periods of intermittent seizures without return to baseline, these exclusions probably do not alter the clinical utility of their data.

Thus, based upon known classifications and using agerelated trends as guidance, an evidence-based evaluation can proceed for the child in SE. In 2006, the quality standards subcommittee of the American Academy of Neurology and the Practice Committee of the Child Neurology Society released a practice parameter to guide the diagnostic assessment of the child with SE, excluding febrile SE [30]. Their review of relevant literature found that lab abnormalities (sodium, glucose, calcium) were present in $6 \%$, low antiepileptic drug (AED) levels in $32 \%$ (although not necessarily the cause of SE), toxicological evidence of ingestion in $3.6 \%$, evidence for an inborn error of metabolism (IEM) in $4.2 \%$, and neuroimaging abnormalities were found in $8 \%$ [30]. It is notable, however, in the series from Singh et al. using more recent data including high quality magnetic resonance imaging (MRI), that imaging yielded a diagnosis in $30 \%$, and directed acute management in $24 \%$ [29•].

For a patient without a known history of epilepsy, evaluation should include basic serum chemistries, including bedside glucose determination and a comprehensive electrolyte panel. Urgent neuroimaging should be performed, preferably MRI, but if unavailable, then with computed tomography. Toxicology and evaluation for IEM is based upon clinical suspicion. However, for patients with known epilepsy who are presenting in SE, repeat neuroimaging may not be needed, unless there are atypical features to the clinical presentation. However, routine electrolyte and AED levels are reasonable.

When a previously normal child in SE has a concurrent fever, the first task is to evaluate and treat for the potential of a primary central nervous system (CNS) infection. If the 
patient proves to not have a CNS infection with a complete evaluation failing to reveal another cause, and they are between 6 months and 5 years old, they fall under the classification of febrile SE. Any febrile illness can provoke a child to have a prolonged febrile convulsion. However, human herpes viruses (HHV) may be an important cause. In a recently published prospective study, of 169 children with febrile status epilepticus, $32 \%$ had serologic evidence of HHV-6 and 7.1\% had serologic evidence of HHV-7 infection [31]. It is not known if high fever caused by these infections or other factors from the virus may cause the SE in susceptible patients, but HHV-6 and HHV-7 appear to have a prominent role for febrile SE in children.

Another special clinical circumstance is SE in the young infant. Consideration of underlying treatable metabolic diseases and vitamin deficiencies should prompt vitamin trials with concurrent EEG, which can be both diagnostic and also therapeutic. Although rare, the early treatment and recognition of pyridoxine dependency, pyridoxal 5'-phosphate dependency, and folinic-acid-dependent seizures are critical for their successful outcome. A proposed algorithm can consist sequentially of pyridoxine $100 \mathrm{mg} \mathrm{IV}$, then (if needed) pyridoxal 5-phosphate $(10 \mathrm{mg} / \mathrm{kg} / \mathrm{dose} \times 2,2 \mathrm{~h}$ apart), and then folinic acid (5 $\mathrm{mg} \times 2,6 \mathrm{~h}$ apart) [32•].

\section{Treatment of Status Epilepticus}

The successful treatment of SE in the PICU begins prior to patient arrival, by both having in place and being familiar with an established scientifically based and practical treatment plan [33]. Having this plan as a protocol promotes the intensive care team's ability to stay one step ahead in the preparation and administration of medications in order to urgently abort SE. This sense of urgency is justified and appropriate, as the time between seizure onset and first-line treatment initiation is inversely correlated with treatment response for both adults [6] and also in children [34]. Delays in initiation of treatment past $30 \mathrm{~min}$ have been shown to be particularly detrimental, although in the PICU, the majority of patients have received treatment prior to their arrival. There is evidence to suggest that the underlying pathophysiology of this is due, at least in part, to decreased sensitivity to gamma-aminobutyric acid (GABA)-A with time due to the down-regulation and internalization of GABA receptors [35].

Furthermore, during the entire continuum of care for the patient with SE, the basics of systemic medical care should be attended to, starting with the maintenance of the patient's airway and breathing, and support of the circulation to include blood pressure. The need for intravenous volume support, the need for antibiotics or antiviral medications for those with suspected infection, and the need for stress steroids for those at risk for adrenal insufficiency should all be assessed simultaneously.

\section{How and What to Treat SE}

Although Class I evidence for pediatric SE is scarce, clinical practice is generally extrapolated from adult Class I data, to include the superiority of lorazepam and diazepam over placebo [36]. The treatment options for pediatric SE are listed in Table 2, which assumes that by the time a patient is in the PICU, intravenous (IV) access has already been established. Furthermore, when patients are transferred into the PICU for treatment of SE, they may have already been treated with first-line medications either in the community or from another inpatient unit, as community treatment has been shown to decrease seizure duration and decrease seizure recurrence [37]. Patients should be quickly assessed as to where along the treatment plan they have progressed, with the next available treatments given as urgently as possible for those continuing to convulse.

\section{Early or Impending SE, First-line}

In the initial stages of SE (5-10 min), first-line therapy includes benzodiazepines, which primarily act as GABA receptor agonists. In particular, those benzodiazepines that can be given in an IV formulation to include lorazepam, diazepam, and midazolam-are preferred for the treatment of SE. However, if IV access is difficult to obtain, alternative methods of administration can be used: for example, intramuscularly, intranasally, or rectally. Due to its longer anticonvulsant effect, lorazepam (12-24 h) is preferred over diazepam and midazolam.

However, if a patient is on chronic maintenance AED for which an IV form is available and for which a recent serum level is available, then this agent may be used to give a partial bolus to increase serum levels as part of firstline therapy. Examples of anticonvulsants in which an IV form is available with serum levels that can be obtained rapidly are phenytoin and valproate. This scenario can also be considered for those patients in which a history can be obtained of missed dosing or admitted noncompliance.

In addition, some children with medically refractory epilepsy may have a vagal nerve stimulator (VNS), for which a magnet can be used to give an additional stimulation. A patient's magnet is often located on their wrist, or attached to a personal item, such as a backpack or wheelchair. When available, the magnet can be swiped slowly once across the VNS which is normally located in the subcutaneous tissue of the left upper chest. As most VNS's are programmed to stimulate for $60 \mathrm{~s}$ with a single swipe, quick repetitive swipes are not necessary, and furthermore 
Table 2 Treatment options for pediatric status epilepticus (SE)

\begin{tabular}{|c|c|c|}
\hline Timing & Treatment & Suggested dosing \\
\hline \multicolumn{3}{|c|}{ Early or impending SE } \\
\hline First-line & $\begin{array}{l}\text { Lorazepam } \\
\text { Diazepam } \\
\text { If VNS, swipe with magnet }\end{array}$ & $\begin{array}{l}0.05-0.1 \mathrm{mg} / \mathrm{kg} \mathrm{IV}, \text { repeat } \times 1 \\
0.2-0.5 \mathrm{mg} / \mathrm{kg} \mathrm{IV}, \text { repeat } \times 1\end{array}$ \\
\hline Second-line & $\begin{array}{l}\text { Fosphenytoin } \\
\text { Phenobarbital } \\
\text { Levetiracetam } \\
\text { Valproate }\end{array}$ & $\begin{array}{l}20-25 \mathrm{PE} / \mathrm{kg} \mathrm{IV} \\
20 \mathrm{mg} / \mathrm{kg} \mathrm{IV} \\
20-30 \mathrm{mg} / \mathrm{kg} \mathrm{IV} \\
20 \mathrm{mg} / \mathrm{kg} \mathrm{IV}\end{array}$ \\
\hline \multicolumn{3}{|l|}{ Refractory SE } \\
\hline \multirow[t]{3}{*}{ First-line } & Midazolam & $\begin{array}{l}\text { Load: } 0.1-0.3 \mathrm{mg} / \mathrm{kg} \text { IV, maintenance: } \\
0.05-0.4 \mathrm{mg} / \mathrm{kg} / \mathrm{h} \mathrm{IV}\end{array}$ \\
\hline & Pentobarbital & $\begin{array}{l}\text { Load: } 2-10 \mathrm{mg} / \mathrm{kg} \text { IV, maintenance: } \\
1-5 \mathrm{mg} / \mathrm{kg} / \mathrm{h} \mathrm{IV}\end{array}$ \\
\hline & Phenobarbital & "High dose", see text \\
\hline \multirow[t]{3}{*}{ Second-line } & Lacosamide & Uncertain pediatric dosing, see text \\
\hline & Propofol & $\begin{array}{l}\text { Load: } 2 \mathrm{mg} / \mathrm{kg} \text { IV, maintenance: } \\
2-5 \mathrm{mg} / \mathrm{kg} / \mathrm{h}\end{array}$ \\
\hline & Topiramate & $\begin{array}{l}\text { Load: } 5-10 \mathrm{mg} / \mathrm{kg} \text { bolus } \mathrm{NG} \text {, maintenance } \\
5-15 \mathrm{mg} / \mathrm{kg} \text { day NG }\end{array}$ \\
\hline Other & $\begin{array}{l}\text { Electroconvulsive therapy } \\
\text { Hypothermia } \\
\text { Ketogenic diet } \\
\text { Immunomodulation } \\
\text { Surgery } \\
\text { Vitamin trials }\end{array}$ & \\
\hline
\end{tabular}

holding the magnet over the VNS is also not recommended, as this will turn the VNS generator off.

\section{Early or Impending SE, Second-line}

If first-line therapies are not successful, then an anticonvulsant with IV formulation for longer-term therapy should be initiated. Fosphenytoin, which acts primarily upon the sodium channel, is used most often in children. It is dosed in phenytoin equivalents (PE), and is usually loaded at 20-25 PE/kg IV, without a maximum dosing. In a recent randomized controlled trial in children, a diazepam-phenytoin combination was just as successful in aborting SE as lorazepam [38]. Although phenytoin is often used in adult SE, in children the prodrug fosphenytoin is preferred. Fosphenytoin administration can be performed at a faster rate than phenytoin (fosphenytoin $150 \mathrm{PE} / \mathrm{min}$ versus phenytoin $25-50 \mathrm{mg} / \mathrm{min}$ ), and fosphenytoin comparatively has lower risks of local caustic effects on tissue, and less risks for hypotension and cardiac dysrhythmias.

Other second-line options include phenobarbital, levetiracetam, and valproate. Phenobarbital is a barbiturate that can be dosed at $20 \mathrm{mg} / \mathrm{kg} \mathrm{IV}$, or in increments of $5-10 \mathrm{mg} / \mathrm{kg}$ to total $20 \mathrm{mg} / \mathrm{kg}$ to start. It is important to be ready to secure a patient's airway when using higher doses of phenobarbital, especially when used following benzodiazepine administration. However, with a secure airway, higher serum levels can be achieved.

Levetiracetam, which has an uncertain mechanism of action, can be loaded at $20-30 \mathrm{mg} / \mathrm{kg} \mathrm{IV}$, and has the advantage of minimal drug-drug reactions and minimal liver metabolism. However, the evidence supporting its use in pediatric SE is confined to case reports and case series. Levetiracetam has been reported to have efficacy in cases of refractory SE [39-41] and also in cases of NCSE [39, $42,43]$. However, in some series, levetiracetam is reported to have poor efficacy for SE [44].

Valproate, which has multiple mechanisms of action, can be loaded at $20 \mathrm{mg} / \mathrm{kg}$. Caution should be practiced in patients with liver disease, and in younger patients ( $<2$-year-old) especially those with an undiagnosed etiology for their developmental delay. Valproate can exacerbate illness in patients with certain abnormalities in energy metabolism such as mitochondrial dysfunction. Retrospective series have found IV valproate to be safe and effective for SE of a wide spectrum of seizure types [45, 46], and a randomized controlled trial in children found valproate to be as effective as diazepam (80\% for valproate vs. $85 \%$ for diazepam), but with less side effects (none for valproate 
versus need for mechanical ventilation in $60 \%$ and hypotension in $50 \%$ for diazepam) [47].

\section{Refractory SE}

Refractory status epilepticus (RSE) occurs when initial attempts to abort SE fail. RSE can be defined as SE lasting over $60 \mathrm{~min}$, which occurs in about $30 \%$ of pediatric SE [29•, 34]. However, clinically, RSE can be defined as SE not responding to at least two anticonvulsant medications. When using a suppressive agent, such as versed or pentobarbital, EEG should be utilized to monitor medication effectiveness with the goal of EEG burst-suppression. In addition, patients at this stage are at least partly encephalopathic, and NCS and NCSE may be difficult to differentiate clinically from a postictal state without EEG support.

\section{Refractory SE, First-line}

The most commonly used medications for RSE are continuous infusions of either midazolam or pentobarbital [48, 49]. Although both can be used effectively [50], pentobarbital has higher rates of adverse effects including hypotension [51]. High-dose phenobarbital can also be used with case series reporting median serum levels of 144 [52] and daily dosing of up to $80 \mathrm{mg} / \mathrm{kg}$ [53].

\section{Refractory SE, Second-line}

Second-line medications for RSE include lacosamide, propofol, and topiramate. Lacosamide enhances the slow activation of sodium channels, and has an IV preparation; however it use and dosing in pediatric SE is not established. A dosing of $25 \mathrm{mg}$ twice a day has been used to successfully treat an 8-year-old boy with RSE [54]. Topiramate, which has multiple proposed mechanisms of action, does not come in an IV preparation, and thus if used in pediatric SE, is administered via nasogastric (NG) tube. Evidence in pediatric RSE consists of case reports and series, and includes loading and maintenance dosing regiments ranging from $5-10 \mathrm{mg} / \mathrm{kg}$ day [55-58]. Continuous propofol infusions, although not uncommonly used in adult $\mathrm{SE}$, are used less in children due to the higher risks of propofol-infusion syndrome in children. Safe and effective use of propofol for RSE has been reported in children [59], although caution and close monitoring should still be used while avoiding higher dosing (over $5 \mathrm{mg} / \mathrm{kg} / \mathrm{h}$ ), and using for the shortest duration possible.

\section{Other Options}

Other treatment options for RSE, for which evidence consists mostly of case reports, that can be considered when initial treatments are ineffective include the ketogenic diet (KD) [60], moderate hypothermia [61], immunomodulation, and electroconvulsive therapy [62]. The KD is a high-fat, low-carbohydrate, adequate protein diet that can be administered as a liquid via NG tube. In a recently published case series of five RSE patients initiated on the $\mathrm{KD}$, after 1 month two patients became seizure-free and the remaining three patients had significant decreases in seizure activity, allowing tapering of other anticonvulsant medications and weaning from prolonged ventilation [60]. Immunomodulation can consist of steroid therapy or intravenous immunoglobulins, and is typically reserved for specific immune-mediated etiologies.

Finally, when medical therapy fails, epilepsy surgery may be considered for carefully chosen candidates. Candidates can include those with focal cortical dysplasia or patients with lateralized lesions, such as Rasmussen's encephalitis [63-65]. In addition, implantation of a vagal nerve stimulator has been reported in the preceding days prior to EEG normalization and being able to wean from suppressive medications in a 6-year-old with RSE [66].

\section{Prognosis}

The goal of urgently treated SE is improved prognosis. In aggressively treated SE, mortality can be low, ranging from 0-9 \% [25, 26, 67, 68], but prognosis is highly dependent upon the etiology $[67,68]$, with deaths occurring mostly within acute symptomatic and progressive neurological etiologies. Morbidity in aggressively treated SE is also low with new neurological deficits occurring in 1-13\% [25, 67, $68]$. Increased morbidity can be associated with increased duration of seizures [67], however in some series this is only for acute symptomatic etiologies [68]. This is consistent with high rates of mortality (30\%) [69], and resultant epilepsy (100\%) [70], in some series of severe prolonged pediatric RSE [71], especially for acute symptomatic etiologies. Overall $11-16 \%$ at 1 year and $18 \%$ at 2 years will have a recurrence of SE [26, 72], but recurring especially in those patients who were not neurologically normal at baseline [72].

Morbidity and mortality, however, are especially rare for febrile SE, with no deaths and no new neurological deficits among a series of 180 patients [73]. However, in a recent study of prospectively obtained data on 199 children with febrile SE, $11.5 \%$ of patients had MRI findings of abnormal increased $\mathrm{T} 2$ signal in one of the hippocampi, which was not present in the controls [74•]. This finding had been reported previously [75], but is now substantiated in a prospective study. Thus it does appear that prolonged febrile seizures can cause focal neuronal injury. However, it is not clear whether these MRI findings are purely due to 
the febrile SE, or whether there was a preexisting risk of injury, as developmental abnormalities, mainly hippocampal malrotation, was noted in $10.5 \%$ of patients with febrile SE versus $2.1 \%$ in the simple febrile seizure control group. The clinical relevance of these findings will hopefully be elucidated with long-term follow-up of these patients; however this data is supportive of the need to aggressively and urgently treat pediatric SE.

An unanswered question at the present is how the diagnosis and treatment of NCS and NCSE in children affects morbidity, mortality, and long-term outcome. Worse outcomes in adults are associated with NCSE [76], however it is not clear if this is influenced more by etiology than as a primary result of NCSE. When NCS or NCSE are suspected, however, expedited diagnosis using EEG and treatment are strongly recommended, realizing that the effect on prognosis remains to be fully understood.

\section{Conclusion}

Status epilepticus is commonly encountered in the PICU. Although most cases manifest obvious clinical signs, PICU patients in SE can sometimes show only subtle signs, necessitating diagnostic support with EEG. Once diagnosed, concurrent evaluation and treatment should be accomplished urgently, and the health-care team should be prepared with a standardized evidence-based plan for treatment. Although outcome is strongly influenced by etiology, urgent treatment is prudent to ensure the best long-term outcome possible. Future long-term prospective studies of the varied types of SE in children encountered in the PICU are needed to help us understand the outcomes of effective diagnosis and treatment of pediatric SE, to include both convulsive and nonconvulsive forms.

Disclosure No conflicts of interest relevant to this article were reported. D.T. Hsieh: "The views expressed in this article are those of the author(s) and do not reflect the official policy or position of Brooke Army Medical Center, the United States Air Force, the United States Army, Department of Defense, or the U.S. Government."

\section{References}

Papers of particular interest, published recently, have been highlighted as:

- Of importance

1. Bell MJ, Carpenter J, Au AK, et al. Development of a pediatric neurocritical care service. Neurocrit Care. 2009;10:4-10.

2. Commission on Epidemiology and Prognosis. International league against epilepsy: guidelines for epidemiologic studies on epilepsy. Epilepsia. 1993;34:592-6.
3. Meldrum BS, Brierley JB. Prolonged epileptic seizures in primates: ischemic cell change and its relation to ictal physiologic events. Arch Neurol. 1973;28:10-7.

4. Lowenstein DH, Bleck T, Macdonald RL. It's time to revise the definition of status epilepticus. Epilepsia. 1999;40:120-2.

5. Shinnar S, Berg AT, Moshe SL, Shinnar R. How long do newonset seizures in children last? Ann Neurol. 2001;49:659-64.

6. Lowenstein DH, Alldredge BK. Status epilepticus at an urban public hospital in the 1980s. Neurology. 1993;43:483-8.

7. Abend NS, Dlugos DJ. Treatment of refractory status epilepticus: literature review and a proposed protocol. Pediatr Neurol. 2008; 38:377-90.

8. Commission on Classification and Terminology of the International League Against Epilepsy. Proposal for revised clinical and electrographic classification of epileptic seizures. Epilepsia. 1981;22:489-501.

9. Chin RF, Neville BG, Peckham C, et al. Treatment of community-onset, childhood convulsive status epilepticus: a prospective, population-based study. Lancet Neurol. 2008;7:696-703.

10. Abend NS, Dlugos DJ, Hahn CD, et al. Use of EEG monitoring and management of non-convulsive seizures in critically ill patients: a survey of neurologists. Neurocrit Care. 2010;12: 382-9.

11. Jette N, Claassen J, Emerson RG, Hirsch LJ. Frequency and predictors of nonconvulsive seizures during continuous electroencephalographic monitoring in critically ill children. Arch Neurol. 2006;63:1750-5.

12. Hosain SA, Solomon GE, Kobylarz EJ. Electroencephalographic patterns in unresponsive pediatric patients. Pediatr Neurol. 2005;32:162-5.

13. Hyllienmark L, Amark P. Continuous EEG monitoring in a paediatric intensive care unit. Eur J Paediatr Neurol. 2007;11: $70-5$.

14. Tay SK, Hirsch LJ, Leary L, et al. Nonconvulsive status epilepticus in children: clinical and EEG characteristics. Epilepsia. 2006;47:1504-9.

15. Saengpattrachai M, Sharma R, Hunjan A, et al. Nonconvulsive seizures in the pediatric intensive care unit: etiology, EEG, and brain imaging findings. Epilepsia. 2006;47:1510-8.

16. McCoy B, Sharma R, Ochi A, et al. Predictors of nonconvulsive seizures among critically ill children. Epilepsia. 2011;52:1973-8.

17. Williams K, Jarrar R, Buchhalter J. Continuous video-EEG monitoring in pediatric intensive care units. Epilepsia. 2011;52: 1130-6.

18. Shahwan A, Bailey C, Shekerdemian L, Harvey AS. The prevalence of seizures in comatose children in the pediatric intensive care unit: a prospective video-EEG study. Epilepsia. 2010;51: 1198-204.

19. Schreiber JM, Zelleke T, Gaillard WD, et al. Continuous video EEG for patients with acute encephalopathy in the pediatric intensive care unit. Neurocrit Care. 2012;17:31-8.

20. • Abend NS, Gutierrez-Colina AM, Topjian AA, et al.: Nonconvulsive seizures are common in critically ill children. Neurology 2011, 76:1071-1077. This is the first prospective study in children to find a high rate of nonconvulsive seizures in critically ill children. Of 100 critically ill children, 46 had nonconvulsive seizures and 19 nonconvulsive status epilepticus.

21. Abend NS, Topjian A, Ichord R, et al. Electroencephalographic monitoring during hypothermia after pediatric cardiac arrest. Neurology. 2009;72:1931-40.

22. • Greiner HM, Holland K, Leach JL, et al.: Nonconvulsive status epilepticus: the encephalopathic pediatric patient. Pediatrics 2012, 129:e748-755. This retrospective study found nonconvulsive status epilepticus in $35 \%$ of 75 pediatric patients with altered mental status in a wide range of inpatient settings, not just the pediatric intensive care unit. 
23. Pakalnis A, Paolicchi J, Gilles E. Psychogenic status epilepticus in children: psychiatric and other risk factors. Neurology. 2000; 54:969-70.

24. Abend NS, Topjian AA, Gutierrez-Colina AM, et al. Impact of continuous EEG monitoring on clinical management in critically ill children. Neurocrit Care. 2011;15:70-5.

25. Hussain N, Appleton R, Thorburn K. Aetiology, course and outcome of children admitted to paediatric intensive care with convulsive status epilepticus: a retrospective 5-year review. Seizure. 2007;16:305-12.

26. Chin RF, Neville BG, Peckham C, et al. Incidence, cause, and short-term outcome of convulsive status epilepticus in childhood: prospective population-based study. Lancet. 2006;368:222-9.

27. Shinnar S, Hesdorffer DC, Nordli DR, et al. Phenomenology of prolonged febrile seizures: results of the FEBSTAT study. Neurology. 2008;71:170-6.

28. Shinnar S, Pellock JM, Moshe SL, et al. In whom does status epilepticus occur: age-related differences in children. Epilepsia. 1997;38:907-14.

29. - Singh RK, Stephens S, Berl MM, et al.: Prospective study of new-onset seizures presenting as status epilepticus in childhood. Neurology 2010, 74:636-642. This is a study of new-onset seizures presenting as status epilepticus, using prospectively obtained data. Neuroimaging with CT and MRI provided a diagnosis in 30\%, a higher yield than previous studies.

30. Riviello JJ Jr. Ashwal S, Hirtz D, et al.: practice parameter: diagnostic assessment of the child with status epilepticus (an evidence-based review). Report of the quality standards subcommittee of the American Academy of Neurology and the Practice Committee of the Child Neurology Society. Neurology. 2006;67:1542-50.

31. Epstein LG, Shinnar S, Hesdorffer DC, et al. Human herpesvirus 6 and 7 in febrile status epilepticus: the FEBSTAT study. Epilepsia. 2012;53:1481-8.

32. - Pearl PL: New treatment paradigms in neonatal metabolic epilepsies. J Inherit Metab Dis 2009, 32:204-213. This is an excellent review discussing neonatal metabolic epilepsies, and offers guidelines for suspecting and diagnosing metabolic epilepsies due to vitamin deficiencies.

33. Glauser TA. Designing practical evidence-based treatment plans for children with prolonged seizures and status epilepticus. J Child Neurol. 2007;22:38S-46S.

34. Eriksson K, Metsaranta P, Huhtala H, et al. Treatment delay and the risk of prolonged status epilepticus. Neurology. 2005;65:1316-8.

35. Goodkin HP, Yeh JL, Kapur J. Status epilepticus increases the intracellular accumulation of GABAA receptors. J Neurosci. 2005;25:5511-20.

36. Alldredge BK, Gelb AM, Isaacs SM, et al. A comparison of lorazepam, diazepam, and placebo for the treatment of out-ofhospital status epilepticus. N Engl J Med. 2001;345:631-7.

37. Alldredge BK, Wall DB, Ferriero DM. Effect of prehospital treatment on the outcome of status epilepticus in children. Pediatr Neurol. 1995;12:213-6.

38. Sreenath TG, Gupta P, Sharma KK, Krishnamurthy S. Lorazepam versus diazepam-phenytoin combination in the treatment of convulsive status epilepticus in children: a randomized controlled trial. Eur J Paediatr Neurol. 2010;14:162-8.

39. Alehan F, Ozcay F, Haberal M. The use of levetiracetam in a child with nonconvulsive status epilepticus. J Child Neurol. 2008;23:331-3.

40. Gallentine WB, Hunnicutt AS, Husain AM. Levetiracetam in children with refractory status epilepticus. Epilepsy Behav. 2009; 14:215-8.

41. Kirmani BF, Crisp ED, Kayani S, Rajab H. Role of intravenous levetiracetam in acute seizure management of children. Pediatr Neurol. 2009;41:37-9.
42. McTague A, Kneen R, Kumar R, et al. Intravenous levetiracetam in acute repetitive seizures and status epilepticus in children: experience from a children's hospital. Seizure. 2012;21:529-34.

43. Trabecca A, Profice P, Costanza MC, et al. Levetiracetam in nonconvulsive status epilepticus in childhood: a case report. J Child Neurol. 2007;22:639-41.

44. Reiter PD, Huff AD, Knupp KG, Valuck RJ. Intravenous levetiracetam in the management of acute seizures in children. Pediatr Neurol. 2010;43:117-21.

45. Uberall MA, Trollmann R, Wunsiedler U, Wenzel D. Intravenous valproate in pediatric epilepsy patients with refractory status epilepticus. Neurology. 2000;54:2188-9.

46. Yu KT, Mills S, Thompson N, Cunanan C. Safety and efficacy of intravenous valproate in pediatric status epilepticus and acute repetitive seizures. Epilepsia. 2003;44:724-6.

47. Mehta V, Singhi P, Singhi S. Intravenous sodium valproate versus diazepam infusion for the control of refractory status epilepticus in children: a randomized controlled trial. J Child Neurol. 2007;22:1191-7.

48. Barberio M, Reiter PD, Kaufman J, et al. Continuous infusion of pentobarbital for refractory status epilepticus in children. J Child Neurol. 2012;27:721-6.

49. Kim SJ, Lee DY, Kim JS. Neurologic outcomes of pediatric epileptic patients with pentobarbital coma. Pediatr Neurol. 2001;25:217-20.

50. Gilbert DL, Gartside PS, Glauser TA. Efficacy and mortality in treatment of refractory generalized status epilepticus in children: a meta-analysis. J Child Neurol. 1999;14:602-9.

51. Holmes GL, Riviello JJ. Midazolam and pentobarbital for refractory status epilepticus. Pediatr Neurol. 1999;20:259-64.

52. Crawford TO, Mitchell WG, Fishman LS, et al. Very-high-dose phenobarbital for refractory status epilepticus in children. Neurology. 1988;38:1035-40.

53. Lee WK, Liu KT, Young BW. Very-high-dose phenobarbital for childhood refractory status epilepticus. Pediatr Neurol. 2006;34:63-5.

54. Shiloh-Malawsky Y, Fan Z, Greenwood R, Tennison M. Successful treatment of childhood prolonged refractory status epilepticus with lacosamide. Seizure. 2011;20:586-8.

55. Akyildiz BN, Kumandas S. Treatment of pediatric refractory status epilepticus with topiramate. Child Nerv Syst. 2011;27: 1425-30.

56. Blumkin L, Lerman-Sagie T, Houri T, et al. Pediatric refractory partial status epilepticus responsive to topiramate. J Child Neurol. 2005;20:239-41.

57. Kahriman M, Minecan D, Kutluay E, et al. Efficacy of topiramate in children with refractory status epilepticus. Epilepsia. 2003;44:1353-6.

58. Perry MS, Holt PJ, Sladky JT. Topiramate loading for refractory status epilepticus in children. Epilepsia. 2006;47:1070-1.

59. van Gestal JP. Blusse van Oud-Alblas HJ, Malingre M, et al.: propofol and thiopental for refractory status epilepticus in children. Neurology. 2005;23:591-2.

60. Nam SH, Lee BL, Lee CG, et al. The role of ketogenic diet in the treatment of refractory status epilepticus. Epilepsia. 2011;52:e181-4.

61. Orlowski JP, Erenberg G, Lueders H, Cruse RP. Hypothermia and barbiturate coma for refractory status epilepticus. Crit Care Med. 1984;12:367-72.

62. Shin HW, O'Donovan CA, Boggs JG, et al. Successful ECT treatment for medically refractory nonconvulsive status epilepticus in pediatric patient. Seizure. 2011;20:433-6.

63. Alexopoulos A, Lachhwani DK, Gupta A, et al. Resective surgery to treat refractory status epilepticus in children with focal epileptogenesis. Neurology. 2005;64:567-70.

64. Ng YT, Kim HL, Wheless JW. Successful neurosurgical treatment of childhood complex partial status epilepticus with focal resection. Epilepsia. 2003;44:468-71. 
65. Schrader DV, Steinbok P, Connolly M. Urgent, resective surgery for medically refractory, convulsive status epilepticus. Eur $\mathbf{J}$ Paediatr Neurol. 2009;13:10-7.

66. De Herdt V, Waterschoot L, Vonck K, et al. Vagus nerve stimulation for refractory status epilepticus. Eur J Paediatr Neurol. 2009;13:286-9.

67. Kravljanac R, Jovic N, Djuric M, et al. Outcome of status epilepticus in children treated in the intensive care unit: a study of 302 cases. Epilepsia. 2011;52:358-63.

68. Maytal J, Shinnar S, Moshe SL, Alvarez LA. Low morbidity and mortality of status epilepticus in children. Pediatrics. 1989;83: 323-31.

69. Sahin M, Menache CC, Holmes GL, Riviello JJ. Outcome of severe refractory status epilepticus in children. Epilepsia. 2001;42: 1461-7.

70. Sahin M, Menache CC, Holmes GL, Riviello JJ. Prolonged treatment for acute symptomatic refractory status epilepticus: outcome in children. Neurology. 2003;61:398-401.

71. Lambrechtsen FA, Buchhalter JR. Aborted and refractory status epilepticus in children: a comparative analysis. Epilepsia. 2008; 49:615-25.
72. Shinnar S, Maytal J, Krasnoff L, Moshe SL. Recurrent status epilepticus in children. Ann Neurol. 1992;31:598-604.

73. Shinnar S, Pellock JM, Berg AT, et al. Short-term outcomes of children with febrile status epilepticus. Epilepsia. 2001;42:47-53.

74. - Shinnar S, Bello JA, Chan S, et al.: MRI abnormalities following febrile status epilepticus in children: the FEBSTAT study. Neurology 2012, 79: 1-7. This is a long-term prospective study of a cohort of 191 children, for which higher rates of hippocampal developmental malformations and increased T2 signal within one of their hippocampi on MRI were found in patients after febrile status epilepticus, compared to the simple febrile seizure control group.

75. VanLandingham KE, Heinz ER, Cavasos JE, Lewis DV. Magnetic resonance imaging evidence of hippocampal injury after prolonged, focal febrile convulsions. Ann Neurol. 1998;43:413-26.

76. Shneker BF, Fountain NB. Assessment of acute morbidity and mortality in nonconvulsive status epilepticus. Neurology. 2003;61: 1066-73. 